\title{
An alternative perspective on the Management of Status Epilepticus
}

\author{
Author Information: \\ ROY G. BERAN \\ M.D (University of NSW, Australia)., \\ F.R.A.C.P (Royal Australian College of GP's, Australia)., \\ F.R.C.P (Royal College of Physicians, England)., \\ F.R.A.C.G.P (Royal Australasian College of Physicians, Australia)., \\ F.A.C.L.M.(Australian College of Legal Medicine, Australia), \\ F.A.F.P.H.M (Royal Australasian College of Physicians, Australia) \\ F.A.C.B.S. (Australasian College of Biomedical Sciences, Australia) \\ B Leg.S. (Macquarie University, Sydney Australia) \\ Grad. Dip. Tert. Ed. (New England University, Armidale, Australia) \\ Grad. Dip. Further Ed. (Adelaide College of Advanced Education, Australia), \\ M.H.L. (University of Sydney, Australia) \\ CONJOINT ASSOCIATE PROFESSOR UNIVERSITY OF NSW, AUSTRALIA
}

PROFESSOR, SCHOOL OF MEDICINE, GRIFFITH UNIVERSITY, BRISBANE, AUSTRALIA

\section{Author's Contact Details:}

Address: Suite 5, level 6, 12 Thomas Street

Chatswood NSW 2067, Australia

Ph: +61 0294114991

Fax: +61 0294114991

roy.beran@unsw.edu.au

(preferred method of communication is e-mail) 


\begin{abstract}
$\underline{\text { Abstract }}$
The definition of status epilepticus (SE) has been reduced from 30 minutes to five minutes and this paper questions if treatment should not be offered before reaching that window. After provision of first aid, benzodiazepines (BDZ) are the initial form of intervention with either nasal or buccal midazolam being favoured for non-professionals. Proper patient supervision, including admission to an intensive care unit for more difficult patients, is endorsed and the need to warn non-professionals of the potential risk of respiratory depression is imperative. The paper criticises the use of phenytoin, as the anti-epileptic medication (AEM) with which to load patients, as it is no longer a first-line AEM and argues in favour of using an AEM which is first-line, such as valproate of carbamazepine, or preferably the AEM that previously proved efficacious in a patient with known epilepsy who was non-compliant. The review discusses alternative routes of administration of AEMs and supports the use of blood level monitoring, as an adjunct to management, to protect against further episodes of SE. The paper touches upon the use of some of the newer AEMs, in the management of SE, and explores treatment strategies which acknowledge that treatment must also include patient education which incorporates techniques to enhance compliance.
\end{abstract}




\section{An Alternative Perspective on the Management of Status Epilepticus}

\section{$\underline{\text { Introduction }}$}

One man's fish (or as the French would say, 'poisson') is another man's poison and yet for too long the concept of 'one meal suits all' pervaded the management of status epileptics (SE). For too long the teaching of medicine has accepted the philosophy that students should ingest, digest and regurgitate on demand. This removes the elements of challenge, question and reappraisal as should be the ethos of all medicine.

With such an introduction one may be excused for presuming that what is to follow represents a 'tongue in cheek' exposé but where better to challenge entrenched behaviour regarding the management of SE than in the Journal entitled Epilepsy and Behaviour? This article will offer an alternative recipe to the management of SE, based on a personal perspective of diagnosis and treatment.

It will suggest greater flexibility which challenges widely accepted dogma while still accepting the underlying concepts. The approach reinstates the ' taste test' which allows epileptologists to modify the treatment algorithm to better accommodate the patient's needs. No apology is offered for the suggested approach, which may be considered avant-garde, as the purpose of this paper is to promote food for thought and to challenge accepted behaviour. 


\section{What is Status Epilepticus?}

$\mathrm{SE}$, as the name implies, is being in the state of having epilepsy rather than having an epileptic seizure. It reflects a time-based diagnosis of being in a seizure rather than a different seizure type and hence there are as many forms of SE as there are seizure types. The diagnosis of SE used to require at least 30 minutes of continuous seizure activity (13) but this timeframe has been reduced to only five minutes before the diagnosis applies and treatment is required as a matter of some urgency (2-4).

This reduction in time is reasonable when one considers that the average convulsive seizure lasts less than a minute (5) and hence five minutes represents a five fold increase of the norm. It makes further sense if one accepts that the choice of 30 minutes appears quite arbitrary.

It has been shown that permanent brain damage may ensue after 20 minutes continuous convulsive activity (6-7). Thus it makes no sense to extend the time required to make the diagnosis of SE by $50 \%$ of the critical window. The choice of five-minutes may still be excessive when one acknowledges that two minutes still represents a $100 \%$ increase on the norm or three minutes duration is a three fold increase. It may prove acceptable to remove the timeframe and to operate on the premise that if a seizure appears prolonged, and one has time to collect the necessary materials to be administered, and the patient is continuing to convulsed, then there is little point to wait an extra 2-3 minutes before 
intervening. The only possible counter-argument might be the potential risk that attaches to the intervention, as will be discussed below.

The concept of a minimum time window for SE is based on the inherent risks of continuous convulsions (6-7). Not all SE carries such risk, as is the case with nonconvulsive SE in which the intervention is often delayed. Non-convulsive SE is often misdiagnosed as aberrant behaviour thereby causing prolonged delay before initiating treatment $(8,9)$. Once non-convulsive SE is suspected an electroencephalograph (EEG) is often necessary for diagnosis, subsequent to which suitable treatment is implemented (10). It should not be ignored that some anti-epileptic medications (AEMs) have a recognised potential to provoke a non-convulsive SE (11) thereby necessitating an increased level of vigilance if such AEMs are used to treat epilepsy.

The one area where debate remains is that related to cluster seizures in which there is no adequate return of consciousness between seizures. In this situation it may be difficult to determine when one seizure stops and the next seizure starts. On this basis, it makes sense to consider such episodes as prolonged seizures with the potential to evoke the same harm as may exist from a single prolonged convulsive episode and hence to treat the cluster as if it were a single seizure and hence SE. 


\section{Immediate Intervention and Seizure Control}

The immediate intervention for SE is predicated by where the SE occurs, the knowledge and experience of those witnessing the SE and the available forms of intervention at the scene. Where SE occurs outside of a medical institution there is no immediate difference between the treatment of SE and that of a single epileptic seizure other than to seek help as a matter of emergency and to transfer the patient to medical care as soon as possible. This in no way abrogates the need for emergency first aid as is applicable to all seizures, namely to maintain airways, to remove foreign bodies (such as teeth or vomitus), to place the patient in a coma/recovery position and to protect the patient from harm $(12,13)$. Similar first aid in SE is also the primary form of intervention should the patient be within a medical facility.

Should the patient be within a medical institution, and first aid has been provided, than it is a simple matter to instigate elementary diagnostic measures while at the same time preparing to provide necessary treatment to stop the seizure activity. Blood samples should be collected to seek a cause and to measure AEM levels, especially in patients known to be treated for epilepsy $(14,15)$. An intravenous (IV) line should be established and the initial IV fluid should be glucose/dextrose based to protect against possible hypoglycaemia in the case of diabetes.

Initial intervention to abort seizures usually relies on benzodiazepines (BDZ) (16-20) and this philosophy is the same whether the patient is within a medical institution or not. The 
New South Wales, Australian case of the Woods (21) placed the onus on the doctor to teach those caring for people with epilepsy to be familiar with the per-rectal (PR) administration of diazepam outside of the medical institution.

The long acting BDZ, lorazepam, is the favoured BDZ in the USA $(18,22)$ but the parental formulation of lorazepam is not universally available and hence cannot be used in countries such as Australia where only the oral preparation is available for the treatment of psychiatric/psychological conditions.

Until recently diazepam was widely advocated as the initial BDZ of choice (23-24). The above cited Woods case (21) highlighted the expectation that PR diazepam should be available outside of the medical environment such that it can be administered to any patient with prolonged seizures (25) although this largely has been superseded by the use of midazolam. Diazepam should not be administered intramuscularly (IM) due to the risk of developing muscle necrosis and sterile absences (26) which limits the route of administration to either PR (available to anyone trained in the technique) and IV (only available to medical and paramedical emergency staff). Studies have compared PR diazepam with midazolam, administered to the buccal mucosa, and have shown comparable efficacy (27) thereby providing a more acceptable form of emergency home intervention to use to abort prolonged seizures.

Midazolam has replaced diazepam as the favoured BDZ in Australia for the treatment of convulsive SE (28-30). It is water soluble which means it can be administered IV (31), 
IM (32), nasally (33) or via the buccal mucosa (27) thereby making it more 'user-friendly' than is PR diazepam and more palatable for use by non-professional carers who have been given suitable instruction as to how and when to administer it $(27,33)$. It must be remembered that midazolam is a relatively short acting BDZ $(34,35)$, hence single administration, other than via continuous IV infusion (36), does not afford the patient long term protection against further seizures. It follows that it should be complemented by a long acting AEM for the management of SE.

A single administration of a BDZ, such as midazolam, may be insufficient to abort a convulsion and a second bolus may be required, outside of the medical establishment, or IV continuous administration may be necessary within the hospital environment, via an IV line, using BDZs such as either midazolam or clonazepam, although some feel that the risk of respiratory depression may restrict such an approach (37).

All BDZ’s carry the potential for respiratory depression (38), a situation that needs to be monitored closely to ensure that the patient continues to breathe, especially if the BDZ is administered outside of a medical environment, such as the family home or hostel. Those administering BDZ's outside the hospital environment must be advised of this risk and must be encouraged to supervise the patient closely with the patient placed on the coma/ recovery position (25), until the patient has regained consciousness. Some argue that the recovery position should only be adopted after the seizure has stopped, to protect against the possibility of dislocating the shoulder (39). Overzealous use of BDZ's, such as 
midazolam, should be discouraged outside the hospital environment with no more than two bolus of midazolam being administered within any 24-hour period.

Where the SE is continuing, despite the emergency use of BDZ's, the patient should be transferred to the intensive care and may require intubation and artificial respiration to maintain airways and to allow adequate monitoring (40). If the use of BDZ has failed to abort the SE there will be need to administer another AEM although by this time the patient should be managed in an intensive care and many will opt to use an anaesthetic agent, such as propofol, a short acting general anaesthetic with rapid onset of action within approximately 30 seconds (41-42). This should only be contemplated within the confines of a high dependency/intensive care environment to allow the intubation, assisted respiration and adequate monitor (43-44).

\section{Beyond the immediate period.}

As has already been stated, most BDZ's have only a short duration action and hence do not provide long term protection against recurrence of seizures and hence a return of convulsive SE (45). It follows that concurrent use of a longer acting AEM, coincidental with the use of the BDZ, is advisable to provide the necessary added protection. The choice of the longer acting AEM should be based on the patient's history, seizure type of and availability within the hospital. 
Many clinicians choose to load the patient with phenytoin (46), irrespective of the fact that they will neither measure the serum level thereof (to confirm adequate loading) nor will they continue to use phenytoin after successful management of SE. Phenytoin is no longer a first line AEM with valproate and carbamazepine considered the gold standards for either focal or generalised epilepsy respectively (47). Acknowledging that phenytoin is no longer a first line AEM (48), clinicians should choose an alternative AEM which is considered to be first line care, making it illogical to load a patient with an AEM that will not be continued into routine care and has the potential to introduce added problems of drug interactions (48), drug withdrawals and confounding potential for unwanted effects.

By far the most common cause for patients to develop SE is a failure of compliance with prescribed AEM for the patients already diagnosed with epilepsy (45). It follows that the logical AEM to reinstate is that AEM which has already proven to be effective in the management of the patient's epilepsy, prior to the lapse in compliance. Once the patient is within the hospital environment, it matters little if the patient becomes toxic, because of the additional administration of the AEM that the patient was taking prior to the SE, as the patient is now under supervised care and control with the availability of intensive monitoring.

Where it is known which AEMs the patient was taking (based on available history) and there is a capacity to measure blood levels, it has already been stated that samples should have been taken, at the time of presentation, to determine the adequacy of compliance 
and which of the AEMs is subtherapeutic, in the situation in which the patient is on polypharmacy.

Not all AEMs are universally available for parenteral administration, as is the case with valproate in Australia. Where IV valproate is available it could, and should, be used (49), where there is no additional information, as it is a broad spectrum AEM and remains a first line agent. Where the parenteral form is not available the potential use of PR administration should not be ignored. The PR delivery of valproate has been accepted for more than three decades as an effective route for administration (50). PR administration of carbamazepine is also effective with rapid absorption and establishment of serum levels (51).

The use of drug level monitoring can greatly enhance the benefits offered by the use of long acting AEM on the basis that there are establish therapeutic windows, especially for longer established AEMs (52), and it makes sense to target the amount administered to achieve an adequate and therapeutic blood level. This may be achieved by the administration of either the IV form of the AEM (where same is available) or PR administration (as already described for valproate and carbamazepine) and then measuring the blood levels of the AEM approximately 30 minutes later. This allows the clinician to determine if the patient has been given an adequate loading and where the level remains below the therapeutic window a second bolus of the AEM may be delivered and the process repeated until adequacy of dosage has been achieved. 
By this means the patient is afforded the additional protection of having a long acting AEM, often the AEM already proven to be effective for the individual patient, which is within the therapeutic range to provide optimal seizure protection with minimal adverse events. It utilises current technology, in a rational and reasonable fashion, to reduce the risk of unnecessary recurrence of SE and allows all who are concerned in the patient's management an added understanding of the amount of AEM present.

\section{Newer agents.}

Some of the newer AEMs, such as levetiracetam, have a parenteral form (53) and are excepted has a broad spectrum, potentially first line AEM (54) but their role in the management of SE remains to be fully established. It seems logical that the administration of IV levetiracetam will play a significant role in the future management of SE (53) but it is too early to make that claim with any certainty. Other agents, such as lacosamide, which also are available in parenteral format, remain within the rubric of experimental AEMs (55) for which only time will tell what place they will have in the treatment of either epilepsy or SE.

Fosphenytoin is an agent that was specifically developed to offer parenteral intervention for convulsive SE (56) but would not be continued after seizure control has been achieved and thus will probably remain at a lower level of acceptability than is afforded other new AEMs. 


\section{Auxiliary intervention}

Having accepted that the most common cause for convulsive SE is noncompliance (19) it follows that adequate management of SE, and protection against future episodes, must include patient education (57). Need for compliance must be emphasised with sufficient vigour to change patient behaviour $(57,58)$. One should not ignore the possible use of aids, such as diaries in which patients record when they take their medication or the involvement of others to help supervise the care where necessary. Even pre-packed dosage containers may be helpful in recalcitrant patients.

If an alternative cause for the SE has been identified, it follows that the identified aetiology must be properly addressed (59), such as the use the antibiotics for intracerebral infection, surgery for space occupying lesions or appropriate intervention for intra-cerebral haemorrhage or stroke. Discussion of these topics is beyond the scope of this paper and will not be considered further.

The final consideration in the management of SE, for the patient who is already diagnosed as having epilepsy, must include a reappraisal of the overall treatment for the epilepsy. Where the AEM(s) used to treat the epilepsy has/have failed to offer seizure control, and compliance has been adequate, there is need to reconsider the AEM to be used but this again falls beyond the scope of this paper. 


\section{$\underline{\text { Conclusion }}$}

This paper has reviewed the management of SE, acknowledging that management involves both the diagnosis and treatment of the condition. It has analysed the timeline necessary for the diagnosis and has raised the question of whether the treatment of SE should commence prior to the five-minute parameter. It has acknowledged the differences that exist between the intervention provided outside, as compared to inside, the hospital environment.

It has appraised the various routes of administration of BDZs, recognising that nasal or buccal midazolam is the favoured form of emergency intervention in the home or hostel, by the non-professional. It cited the Australian case of Woods, in which the use of such approaches has become the responsibility of the doctor to teach the carers of patients with epilepsy. It challenged the loading of patients with phenytoin, rather than a first line AEM, and the failure to utilise drug level monitoring as part of the routine management of SE. In addition it provided consideration of alternative routes of administration of AEM, such as the PR administration of valproate or carbamazepine and touched upon contemplation of the newer AEM, such as levetiracetam.

The purpose behind this paper has been to challenge accepted dogma and to offer an alternative approach which has the potential to enhance patient care and limit the risk of harm. 


\section{Acknowledgements:}

A special mention must go to Annaliese Hurley for her help with the preparation of this paper. 


\section{References:}

1. Waterhouse EJ, deLorenso RJ. Status Epilepticus in Older Patients: Epidemiology and Treatment Options. Drugs \& Aging 2001; 18 (2): 133-142.

2. Scott, RC, Surtees RAH, Neville BGR. Status epilepticus: pathophysiology, epidemiology, and outcomes. Arch Dis Child 1998; 79: 73-7.

3. Lowenstein DH. It's time to revise the definition of status epilepticus. Epilepsia 1999; 40:120-2.

4. Waterhouse EJ. Status Epilepticus. Current Treatment Options in Neuology 2002; 4: 309-17.

5. Prego-Lopez M, Devinsky O. Evaluation of a first seizure - Is it epilepsy? Postgraduate Medicine 2002; 111 (1): 34-48

6. Nevander G, Ingvar M, Auer R, Siesjo BK. Status epilepticus in welloxygenated rats causes neuronal necrosis. Ann Neurol. 1985;18:281-290.

7. Treatment of convulsive status epilepticus: Recommendations of the Epilepsy Foundation of America's Working Group on Status Epilepticus. JAMA 1993;270:854-9. 
8. Walker MC. Diagnosis and Treatment of Nonconvulsive Status Epilepticus. CNS Drugs 2001; 15 (12): 931-9.

9. Krumholz A. Epidemiology and evidence for morbidity of nonconvulsive status epilepticus. J Clin Neurophysiol 1999; 16(4): 314-22.

10. Kaplan PW. Assessing the Outcomes in patients with Nonconvulsive Status Epilepticus: Nonconvulsive Status Epilepticus is Underdiagnosed, Potentially Overtreated, and Confounded by comorbidity. J Clin Neurophysiol 1999; 16(4): $341-52$.

11. Kellinghaus C. Dziewas R. Ludemann P. Tiagabine-related non-convulsive status epilepticus in partial epilepsy: three case reports and a review of the literature. Seizure 2002; 11:243-9.

12. Tasker RC. Emergency treatment of acute seizures and status epilepticus. Arch Dis Child 1998; 79: 78-83.

13. Staneveld E, Staneveld AA. Status Epilepticus: Current concepts and management. Can Fam Phy. 2000; 46: 1817-23. 
14. Treiman DM. Convulsive status epilepticus. Curr Treat Opin in Neuol. 1999; 1: $359-69$.

15. Jagoda A, Colucciello SA. Seizures: Accurate diagnosis and effective treatment. Emerg Med Prac. 2000; 2(10): 2-24.

16. Treiman DM. Pharmacokinetics and chemical use of benzodiazepines in the management of status epilepticus. Epilepsia 1989: 30 (suppl 2): S4-10.

17. Bassin S. Smith TL. Bleck TP. Clinical Review: Status Epilepticus. Critical care 2002; 6: 137-42.

18. Cock HR. Schapria AHV. A comparison of lorazepam and diazepam as intial therapy in convulsive status epilepticus. Q J Med 2002; 95: 225-31.

19. Marik PE. Varon J. Management of Status Epilepticus. Chest 2004; 126: 58291.

20. Smith BJ. Treatment of Status Epilepticus. Neurol Clin 2001; 19: 347-69.

21. Woods v Lowns \& Procopis, Court of Appeal, 5/2/96 ref; 40094/1995. 
22. Scott RC. Neville BGR. Pharmacological management of convulsive status epilepticus in children. Dev Med Child Neurol 1999; 41:207-10.

23. Lowenstein DH. Status epilepticus. West J Med 1998. 168 (4) 263.

24. Lockey AS. Emergency department drug therapy for status epilepticus in adults. Emerg. Med. J. 2002; 19: 96-100.

25. O'Sullivan C. Harvey S. The use of rectal diazepam for the treatment of prolonged convulsions in children: Consumer Comments. Aust Prescr 1998; 21: 35-6.

26. Bongard FS, Sue DY. Current Critical Care Diagnosis \& Treatment. $2^{\text {nd }}$ Edition. New York: Lange Medical Books / McGraw-Hill; 2002, p. 119.

27. Scott RC, Besag FMC, Veville BGR. Buccal midazolam and rectal diazepam for treatment of prolonged seizures in clildhood and adolescence: a randomised trial. Lancet 1999; 353: 623-6.

28. Scott RC, Neville BGR. Pharmacological management of convulsive status epilepticus in Children. Developmental Medicine and Child Neurology 1999; 41: 207-10. 
29. Galvin GM, Jelinek GA. Midazolam: an effective intravenous agent for seizure control. Arch Emerg Med 1987; 4(3): 169-72.

30. Durham D. Management of Status Epilepticus. Critical care and Resuscitation 1999; 1: 344-53.

31. Lal Koul R. Raj Aithala G. Chacko A. Joshi R. Seif Elbualy M. Continuous midazolam infusion as treatment of status epilepticus. Arch Dis Child 1997 ;76: 445-448.

32. Towne AR. DeLorenzo RJ. Use of intramuscular midazolam for status epilepticus. J Emerg Med 1999; 17: 322-8.

33. Lahat E, Goldman M, Barr J, Bistritzer T, Berkovitch M. Comparison of intranasal midazolam with intravenous diazepam for treating febrile seizures in children: prospective randomised study. BMJ 2000; 321: 83-86.

34. Blumer JL. Clinical Pharmacology of Midazolam in Infants and Children. Clin Pharmacokinetics 1998; 35(1): 37-47

35. Brevoord JC, Joosten KF, Arts WF, van Rooij RW, de Hoog M. Status Epilepticus: Clinical analysis of a treatment protocol based on Midazolam and Phenytoin. J. Child. Neurol. 2005; 20(6): 476-81. 
36. Lal Koul R, Raj Aithala G, Chacko A, Joshi R, Seif Elbualy M. Continuous midazolam infusion as treatment of status epilepticus. Arch Dis Child 1997; 76: $445-8$

37. Shorvon S. The management of status epilepticus. J Neurol Neurosurg Psychiatry 2001; 70 (suppl 2): ii22-ii27.

38. Walker M. Status Epilepticus: an evidence based guide. BMJ 2005: 331; 673-7.

39. DeToledo JC, Lowe MR. Seizures, lateral decubitus, aspiration, and shoulder dislocation - Time to change the guidelines? Neurology 2001; 56: 290-1.

40. Howard RS, Kullmann DM, Hirsch NP. Admission to neurological intensive care: who, when, and why? J Neurol Neurosurg Psychiat 2003; 74: 2-9

41. Rosenow F, Arzimanoglou A, Baulac M. Recent Development in Treatment of Status Epilepticus: A review. Epileptic Disord. 2002; 4( Suppl 2): S1 1-11.

42. Grant IS, Andrews PJD. Neurological support. BMJ 1999; 319(7202): 110-3.

43. Tasker RC. Emergency treatment of acute seizures and status epilepticus. Arch Dis Child 1998; 79: 78-83. 
44. Webb T, Cockerell C. Current role of drug treatment in the management of epilepsy. Prescriber 2004; 15(3): 54-60.

45. Marik PE, Varon J. The Management of Status Epilepticus. Chest 2004; 126: 582-91.

46. Walker M. Status epilepticus: an evidence based guide. BMJ 2005; 331: 673-7

47. Hart YM. Should first-line treatment of epilepsy differ for males and females? J R Coll Physicians Edinb. 2003; 33(suppl 11): 11-21.

48. Nation RL, Evans AM, Milne RW. Pharmacokinetic drug interactions with phenytoin (Part 1). Clin Pharmacokinet. 1990; 18(1): 37-60.

49. Kanner AM. The Pharmacology of Parenteral Valproate. Epilepsy Curr. 2003; 3(3): 109-11.

50. Vajda FJ, Symington GR, Bladin PF. Rectal valproate in intractable status epilepticus. Lancet 1977; 1(8007): 359-60.

51. Personal experience of author 
52. Eadie MJ., Therapeutic drug monitoring - antiepileptic drugs. Br J Clin Pharmacol 1998; 46: 185-93.

53. Patel NC, Landan IR, Levin J, Szaflarski J, Wilner AN. The use of levetiracetam in refractory status epilepticus. Seizure 2006; 15: 137-141.

54. Beyenburg S, Bauer J, Reuber M, New drugs for the treatment of epilepsy: a practical approach. Postgrad Med J. 2004; 80: 581-7.

55. Kenney P, Simpson J, Koch B. SP768 study group. A multi-center randomized double blind placebo controlled trial to assess the efficacy and safety of lacoamide (200, 400 and $600 \mathrm{mg} /$ day) in subjects with painful distal diabetic neuropathy. Program and abstracts of the 25th Annual Scientific Meeting of the American Pain Society; May 3-6, 2006; San Antonio, Texas. Poster 774.

56. Curry W, Kulling D. Newer antiepileptic drugs: gabapentin, lamotrigine, felbamate, topiramate and fosphenytoin. Am F Phys 1998; 57: 513-20.

57. Leppik IE. How to get patients with epilepsy to take their medication: The problem of noncompliance. Postgrad Med 1990; 88: 253-6.

58. Senanayke N, Peiris H. Mortality related to convulsive disorders in a developing country in Asia: trends over 20 years. Seizure 1995; 4(4): 273-7. 
59. Shorvon SD, Walker MC. The treatment of tonic clonic status epilepticus. In: Duncan JS, Sisodiya SM, Smalls JE (eds) Epilepsy 2001: From Science to Patient $8^{\text {th }}$ Edition. Edenbridge, Meritus Communications 2001. pp 331-336. 